DRAFT VERSION JULY 31, 2021

Typeset using LATEX twocolumn style in AASTeX61

\title{
CHARACTERIZING THE MOTION OF SOLAR MAGNETIC BRIGHT POINTS AT HIGH RESOLUTION
}

\author{
SAMuel J. VAN KoOten ${ }^{1}$ AND STEVEn R. CRANMER ${ }^{1}$
}

${ }^{1}$ Department of Astrophysical and Planetary Sciences, University of Colorado, Boulder, Colorado, 80309, USA

\begin{abstract}
Magnetic bright points in the solar photosphere, visible in both continuum and G-band images, indicate footpoints of kilogauss magnetic flux tubes extending to the corona. The power spectrum of bright-point motion is thus also the power spectrum of Alfvén wave excitation, transporting energy up flux tubes into the corona. This spectrum is a key input in coronal and heliospheric models. We produce a power spectrum of bright-point motion using radiative magnetohydrodynamic simulations, exploiting spatial resolution higher than can be obtained in present-day observations, while using automated tracking to produce large data quantities. We find slightly higher amounts of power at all frequencies compared to observation-based spectra, while confirming the spectrum shape of recent observations. This also provides a prediction for observations of bright points with DKIST, which will achieve similar resolution and high sensitivity. We also find a granule size distribution in support of an observed twopopulation distribution, and we present results from tracking passive tracers which show a similar power spectrum to that of bright points. Finally, we introduce a simplified, laminar model of granulation, with which we explore the roles of turbulence and of the properties of the granulation pattern in determining bright-point motion.
\end{abstract}

Keywords: convection — magnetohydrodynamics (MHD) — Sun: granulation — Sun: photosphere — waves 


\section{INTRODUCTION}

The surface of the Sun is the upper boundary of its convective zone, where convective cells called granules appear on spatial scales of $1 \mathrm{Mm}$ and change on timescales of 10 minutes. In the dark lanes between granules, bright, high-contrast structures are found, ranging from isolated points to extended structures wrapping around the granules. These features, called "G-band bright points," "network bright points," "magnetic bright points," "magnetic bright features," "inter-granular bright points," and "filigree", will be referred to simply as bright points in this paper. Small, isolated bright points are of order $0.1 \mathrm{Mm}$ across and are visible for a few minutes at a time. Bright points are the footpoints of kilogauss-strength magnetic flux tubes extending into the corona. In bright points, the concentrated magnetic flux reduces gas pressure, lowering the optical-depth-unity surface below that of the surrounding areas by about $350 \mathrm{~km}$ (Carlsson et al. 2004; Nordlund et al. 2009). This lower depth corresponds to a higher temperature (an $\sim 800 \mathrm{~K}$ difference), producing enhanced brightness relative to the surrounding downflow lanes. This effect is visible in continuum imaging with sufficient resolution, but the contrast between bright points and even the brightest granular areas is significantly enhanced in wavelengths such as the $430.5 \mathrm{~nm}$ G-band. The $\mathrm{G}$-band is dominated by lines from $\mathrm{CH}$, a molecule whose abundance is significantly reduced at the optical bottom of bright points through dissociation (Steiner et al. 2001). This reduced $\mathrm{CH}$ opacity allows more light to shine through from the continuum below, producing significantly enhanced Gband contrast.

The dynamics of bright points are important to study as a compelling potential source of coronal heating. Bright points move due to buffeting from the convective churning of the photosphere (Berger \& Title 1996). This motion transverse to the emerging flux tubes excites transverse magnetohydrodynamic (MHD) waves in the tubes. These waves propagate up to the corona, where they are thought to turbulently dissipate, depositing heat. Thus the power spectrum of bright-point motion is directly related to the power spectrum of these transverse waves. This wave-heating power spectrum serves as an important boundary condition for models of transverse MHD wave propagation in the solar environment (e.g. Cranmer \& van Ballegooijen 2005) and thus informs models of MHD-wave based coronal heating as well as models of the heliosphere.

A number of observations have been made of bright points. Approaches to understanding their motion include the use of passive tracers that follow the gradient of magnetic intensity images (van Ballegooijen et al. 1998), an approach which can handle extended structures; and the tracking of features identified by hand in individual frames (Nisenson et al. 2003; Chitta et al. 2012), which excels in directly track- ing isolated bright points of limited size. More recent studies improve this latter approach with automatic identification of bright points, producing much larger datasets (Utz et al. 2010; Abramenko et al. 2011; Keys et al. 2011; Yang et al. 2014).

Observational determinations of the bright-point power spectrum are limited by the resolution and cadence of the observations. Seeing conditions and pointing jitter can also limit the ability to track bright points accurately over long durations. In this paper, we use high-resolution simulations of the photosphere to produce a power spectrum of brightpoint motion tracked at higher spatial resolution than has been done before. This serves as a prediction of the observational bright-point power spectrum that the Daniel K. Inouye Solar Telescope (DKIST; Tritschler et al. 2016) will obtain with unparalleled spatial and temporal resolution. We also introduce a simplified model of granulation, which allows investigation of the interplay between bright-point dynamics and the complex properties of the granulation pattern. Section 2 will describe our analysis of the high-resolution MURaM simulation. Section 3 will introduce our simplified, laminar ROUGH model. Section 4 will present our results, and Section 5 will summarize our conclusions.

\section{MURaM SIMULATIONS}

\subsection{Introduction}

Rempel (2014) uses MURaM, software for performing radiative MHD simulations, to simulate the upper convective zone and photosphere. The version of MURaM used is extensively modified from the original of Vögler et al. (2005). In this paper we analyze the results of a run with a domain size of $24.576 \times 24.576 \times 7.680 \mathrm{Mm}^{3}$, at a horizontal resolution of $16 \mathrm{~km}$ per pixel. The data set covers $8280 \mathrm{~s}$, or 2.3 hours, of time at a cadence of one snapshot every 20.7 seconds (for 401 total snapshots). The simulation run begins with an imposed mean vertical magnetic field of $30 \mathrm{G}$ (as discussed in Section 4.3 of Rempel (2014)), but is otherwise identical to the "O16bM" described in the paper's Table 1 . The imposed flux is quickly swept into the downflow lanes, resulting in an enhancement of kilogauss features. The top boundary is positioned $1.5 \mathrm{Mm}$ above the $\tau=1$ surface of the photosphere, allowing a convective region depth of $6.2 \mathrm{Mm}$. From this simulation we take the $\tau=1$ surface as our solar-observation analogue. Within this slice, we use the upward-pointing white light intensity and the three components of both plasma velocity and magnetic field. A sample patch of the simulation is shown in Figure 1.

\subsection{Analysis Algorithms}

From these MURaM simulations, we extracted granule size distributions, the motion paths of passive tracers, and the motion paths of automatically-identified bright points. This 

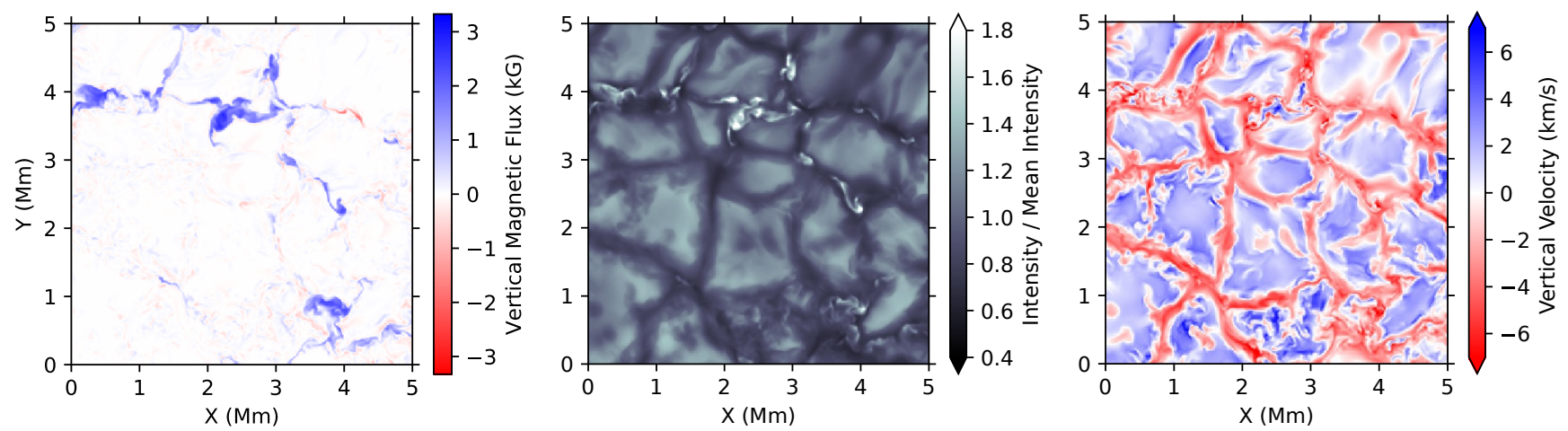

Figure 1. A common $5 \times 5 \mathrm{Mm}$ portion of the Rempel (2014) MURaM simulation showing, from left to right, vertical magnetic flux, whitelight intensity, and vertical plasma velocity at the beginning of the analyzed time range (time stamp 040000). Over the full frame, peak values for $I / I_{\text {mean }}$ are near 2.75 , peak values for $B_{z}$ are near $3.3 \mathrm{kG}$, and peak values for $V_{z}$ are near $9.9 \mathrm{~km} \mathrm{~s}^{-1}$ and $-11.9 \mathrm{~km} \mathrm{~s}$. (The full $24.5 \times 24.5 \mathrm{Mm}$ simulation is used in this paper.)

section describes the algorithms used for collecting this information, while the data products themselves are analyzed in Section 4.

For the identification of granules, observers such as Abramenko et al. (2012) use intensity from non-spectrographic data to segment granules. With the MURaM data, we segment granules based on vertical velocity instead, using just a positive-negative threshold. While deviating from observational approaches, this produces very similar results with a simple, more direct technique.

We track passive, point-like test particles, called "corks," as one way to analyze the plasma flows in the MURaM data. We begin with a uniformly-spaced grid of 4,900 corks. (We find the power spectrum of cork motion to be very insensitive to the initial arrangement.) Once initially placed and released, corks move according the horizontal portion of the velocity vector. We linearly interpolate in time to a timestep of $4 \mathrm{~s}$ when propagating corks, to ensure corks in small, highplasma velocity regions (e.g. turbulent whirls) do not maintain that high velocity after quickly exiting the region. However, when computing power spectra, we sample the cork velocity every $20 \mathrm{~s}$ in order to avoid exceeding the Nyquist rate of the data. With a timestep of $4 \mathrm{~s}$, a pixel size of $16 \mathrm{~km}$, and typical plasma velocities of $\sim 2 \mathrm{~km} \mathrm{~s}^{-1}$, a cork stays near its initial location during a timestep. Cork locations are stored to floating-point precision, and the velocity field is interpolated spatially from the four nearest grid points. Corks are allowed to travel through the periodic boundaries of the simulation. Cork velocities used for computing power spectra are those read out of the interpolated velocity field at cork locations.

For tracking bright points themselves, we use an algorithm that first identifies features within a given frame, following Feng et al. (2012), and then links identified features between frames, following Yang et al. (2014). The intra-frame algorithm keys in on the fact that bright points are small and are characterized by high contrast (they are "bright" relative to their immediate surroundings, but not always in absolute terms-especially in continuum images). In each step, we err toward preventing false positive detections at the cost of more false negatives, since there is such a wealth of bright points available to be detected.

The single-frame stage begins by identifying "seed" pixels in a white-light intensity map. A discrete analogue of the Laplacian is calculated by subtracting from each pixel's intensity the mean intensity of the eight surrounding pixels. A local maximum in intensity will produce a significantly positive value, and this indicates a likely bright-point center. We use a threshold of three standard deviations from the mean in the Laplacian to identify a set of likely centers.

Next, the set of candidate pixels is dilated to include pixels that both exceed a lower threshold and neighbor an alreadyselected pixel (including neighbors along a diagonal). We repeat this dilation process three times, and the threshold for inclusion in this stage is a positive Laplacian value. This process expands the set of selected candidate pixels from the "seed" pixels, which are quite likely to be in bright points, to a nearby cloud of pixels whose likelihood of being in a bright point is enhanced by virtue of being near a pixel already considered a candidate. Since the structure of a bright point tends to be a decrease in intensity from a local maximum to low values at the edge, the use of the Laplacian > 0 threshold aims to ensure that the dilation includes pixels within the bright point but stops expanding where it reaches the edge of the bright point. At this point, each contiguous block of selected pixels can be identified and labeled as a unique candidate bright point. An example of such a bright point is shown in Figure 2.

The next step is to remove as false positives local intensity maxima inside granules. After the previous three rounds of dilation, these local maxima will produce identified features surrounded by further bright pixels. True bright points, however, tend to be mostly or entirely included within the identified set of pixels at this stage, so the identified feature will be surrounded by dark intergranular lane pixels. (A bright-point 


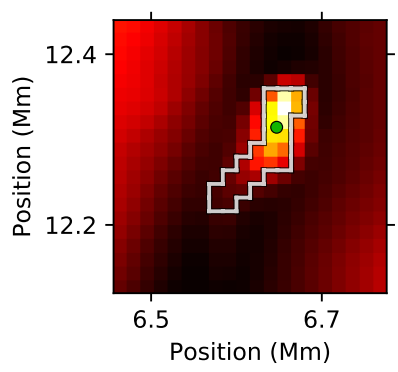

Figure 2. Intensity (left) and vertical magnetic field (right) for an example identified bright point. The gray line marks the borders of the identified region, while the green dot marks the intensityweighted centroid. Identification is on the basis of intensity, and the vertical magnetic field shows the expected concentration.

diameter is of order $100 \mathrm{~km}$, and a pixel in this simulation is $16 \mathrm{~km}$ across. Three rounds of dilation allow a maximum expansion of $16 \times 3=48 \mathrm{~km}$ or one bright-point radius, meaning a seed pixel at the center can be expanded to cover a typical bright point. Often more than one seed pixel will be identified within a feature, allowing features on the large end of the spectrum to easily be captured.) We compute the fraction of a feature's neighboring pixels which would be added to the feature if a fourth round of dilation occurred (still using the Laplacian $>0$ threshold). We reject as false positives any features for which more than $20 \%$ of these new neighboring pixels have positive Laplacians.

We apply two more criteria to ensure a high-quality selection of bright points. First, we reject features within four pixels of another feature. This ensures the analysis is of bright points that are cleanly and unambiguously separated from other bright points. We reject as short-lived transients features below 4 total pixels in area (36 km equivalent diameter). We also reject features above 110 total pixels in area (189 km equivalent diameter) or above 20 pixels $(320 \mathrm{~km})$ across (as determined by finding the diagonal of the smallest rectangle to contain the feature), to ensure identified features are not so large or extended that their motion cannot be represented by the motion of their centroid. (This necessarily eliminates from consideration the long chains of connected bright points that can occur in regions of strong background magnetic flux; see Section 5.)

This completes the intra-frame identification step. Each frame now has a set of uniquely-identified features, but these features must be linked in the temporal dimension for any analysis. This linking step, following Yang et al. (2014), is short: if a feature in frame $n$ overlaps, by at least one pixel, the location of a feature in frame $n-1$, the two features are considered to be the same. This is supported by observations (e.g. Chitta et al. 2012; Nisenson et al. 2003), which find typical bright-point sizes of 100-150 km and typical velocity distributions with maxima around $7 \mathrm{~km} \mathrm{~s}^{-1}$. In the simulation's 20-second time steps, a bright point would move a

maximum of $140 \mathrm{~km}$ (with most bright points moving much less), ensuring that most bright points will overlap with their prior position. In situations where a feature in frame $n$ overlaps multiple features in frame $n-1$ (e.g. two bright points merge), or where multiple bright points in frame $n$ overlap one feature in frame $n-1$ (e.g. a bright point splits in two), linkage is ambiguous and is not attempted-instead, the feature(s) in frame $n$ are considered newly-found features. Once linking is completed across all frames, we reject bright points with lifetimes under 5 frames (1.6 minutes) as a further guard against transient features.

The result of this algorithm is a set of bright-point identification numbers, with each number corresponding to a set of coordinates within each frame of the bright point's lifespan.

\section{ROUGH MODEL}

\subsection{Introduction}

In an attempt to accurately and efficiently capture the laminar portion of the solar granulation pattern, we developed a new Monte Carlo-based model called ROUGH (Random, Observationally-motivated, Unphysical, Granulation-based Heliophysics). ROUGH simulations are highly phenomenological, and the properties of the simulation are drawn from observations of solar granulation. The model aims to produce a reasonable representation of granulation at a single slice of constant height while offering very direct control over the properties of that granulation pattern.

In a ROUGH simulation, a set number of granule centers are randomly distributed over a region of space and throughout a given time span. Granule centers are assigned a horizontal drift speed, drawn from a Gaussian distribution of zero mean and width $0.25 \mathrm{~km} \mathrm{~s}^{-1}$ (Roudier et al. 2012), as well as a drift direction drawn from a uniform distribution. Each center is also assigned a lifetime randomly drawn from the lifetime distribution of Del Moro (2004). We use the exponential fit to lifetimes $>5$ minutes $\left(e^{-t / 2.7 \mathrm{~min}}, t>5 \mathrm{~min}\right)$, as this produces excellent agreement between simulated and observed granulation movies. This produces a mean lifetime of $7.7 \mathrm{~min}$. At the end of a granule's lifetime, a granule can fade away or it can fragment. Lemmerer et al. (2017) show that the majority of granules fragment into two or more child granules. We give each simulated granule a $70 \%$ chance of fragmenting into exactly two children. The child granules each have new lifetimes drawn again from the Del Moro (2004) distribution.

Each granule center is taken as a point source of radiallydiverging, horizontal flow with a time-dependent amplitude of

$$
V_{r}(t)=V_{\max } e^{-\left(\left(t-t_{\text {mid }}\right) / \delta\right)^{4}},
$$

where $t_{\text {mid }}$ is the midpoint of the granule center's lifetime and $\delta$, set to a third of the granule's lifetime, determines the falloff rate with time. $V_{\max }$ is set to $5.1 \mathrm{~km} \mathrm{~s}^{-1}$ to match the 
integrated ROUGH power spectrum to the integrated MU$\mathrm{RaM}$ cork-tracking power spectrum. The radial flow is given a spatial dependence of

$$
V_{r}(r, t)=V_{r}(t) \frac{r}{r_{0}} \frac{1}{\left(1+\left(r / r_{0}\right)^{a}\right)^{b}},
$$

where $r_{0}=500 \mathrm{~km}, a=4$ and $b=1 / a$ determine the shape of the function, and $r$ is the horizontal distance from a location to the center of the granule. This functional form is chosen to match the observed granulation pattern (see also Simon \& Weiss 1989). At each pixel in the simulation, the horizontal velocity is taken to be the velocity corresponding to the largest value of $V_{r}(r, t) / r$ produced at that pixel by any one granule center. In other words, each granule center has a "sphere of influence" in which it is both the sole producer and the strongest possible producer of horizontal flow. $V_{r} / r$ is used for this step rather than $V_{r}$ itself to flatten the initial ramp-up from $V_{r}(r=0)=0$ to the function's maximum value.

At this point, the modeled granulation pattern will have discontinuous transitions at granule boundaries. Gaussian smoothing with a $1 / e$ full-width of $317 \mathrm{~km}$ is applied to the horizontal velocities to produce realistic inter-granular lanes of finite size. An example of the resulting granulation pattern is shown in Figure 3.

As a final step, vertical velocities are calculated by mass conservation in an assumed hydrostatic density profile, using

$$
V_{z}=H_{\text {eff }}\left(\frac{d V_{x}}{d x}+\frac{d V_{y}}{d y}\right)
$$

(see Simon \& Weiss 1989), where the effective scale height $H_{\text {eff }}$, defined in terms of the pressure and vertical velocity scale heights by $1 / H_{\text {eff }}=1 / H_{P}+1 / H_{V_{z}}$, is set to $75 \mathrm{~km}$ computed from values of $H_{V_{z}} \approx 150 \mathrm{~km}$ (Oba et al. 2017) and $H_{P} \approx 150 \mathrm{~km}$.

ROUGH cannot contain explicit bright points, so instead we analyze the horizontal flow fields using passive tracers ("corks"). A cork is placed initially at the center of the simulation domain. At each time step, the horizontal velocity at the cork's location is calculated by a two-dimensional interpolation from the four nearest pixels, and the cork moves with that velocity until the next timestep. The cork's location is not constrained to discrete pixel locations, but is calculated and saved to full numeric precision. When calculating power spectra, we remove the initial portion of each cork's path, up until the cork first reaches a pixel with a downward vertical velocity. This is more representative of actual bright points, though we find that doing so produces little change in the final power spectrum. An example cork motion track is shown in Figure 3.

The ROUGH model contains a number of adjustable parameters that control the granulation pattern, and varying
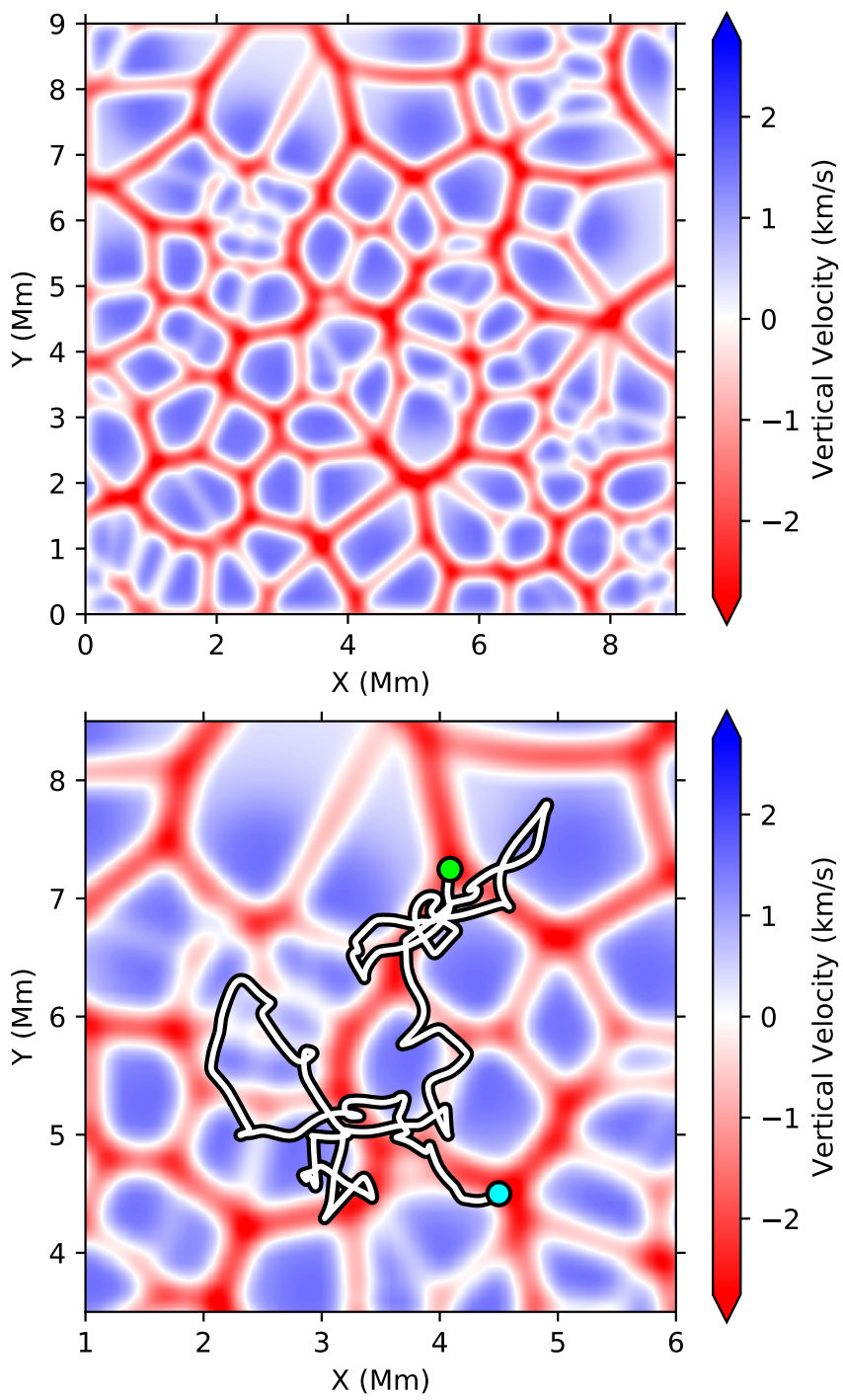

Figure 3. Sample granulation pattern produced by our ROUGH model. On the top, the full simulation domain. On the bottom, a magnified portion showing the motion track of a single cork over 166 minutes. The cork moved from the cyan dot on the bottom to the green dot on the top. The granulation pattern shown in both plots is that of the final frame.

those parameters yields physical insight. We find that the cork power spectra are insensitive to the granule drift velocity distribution, consistent with the fact that granule drift is slow compared to granular flow velocities and to the timescale of change in the granulation pattern. We find increased power in cork motion when the horizontal plasma velocities are increased, with the integrated power spectrum following a $\propto V_{\max }^{2}$ scaling for moderate changes in velocity (reflecting the nature of kinetic energy). We also find that total power is decreased when the granule emergence rate (per unit area, per unit time) is increased or when the granule scale size $r_{0}$ (Equation 2) is increased. Increasing the emergence rate increases the number of granules and thus the fraction of 

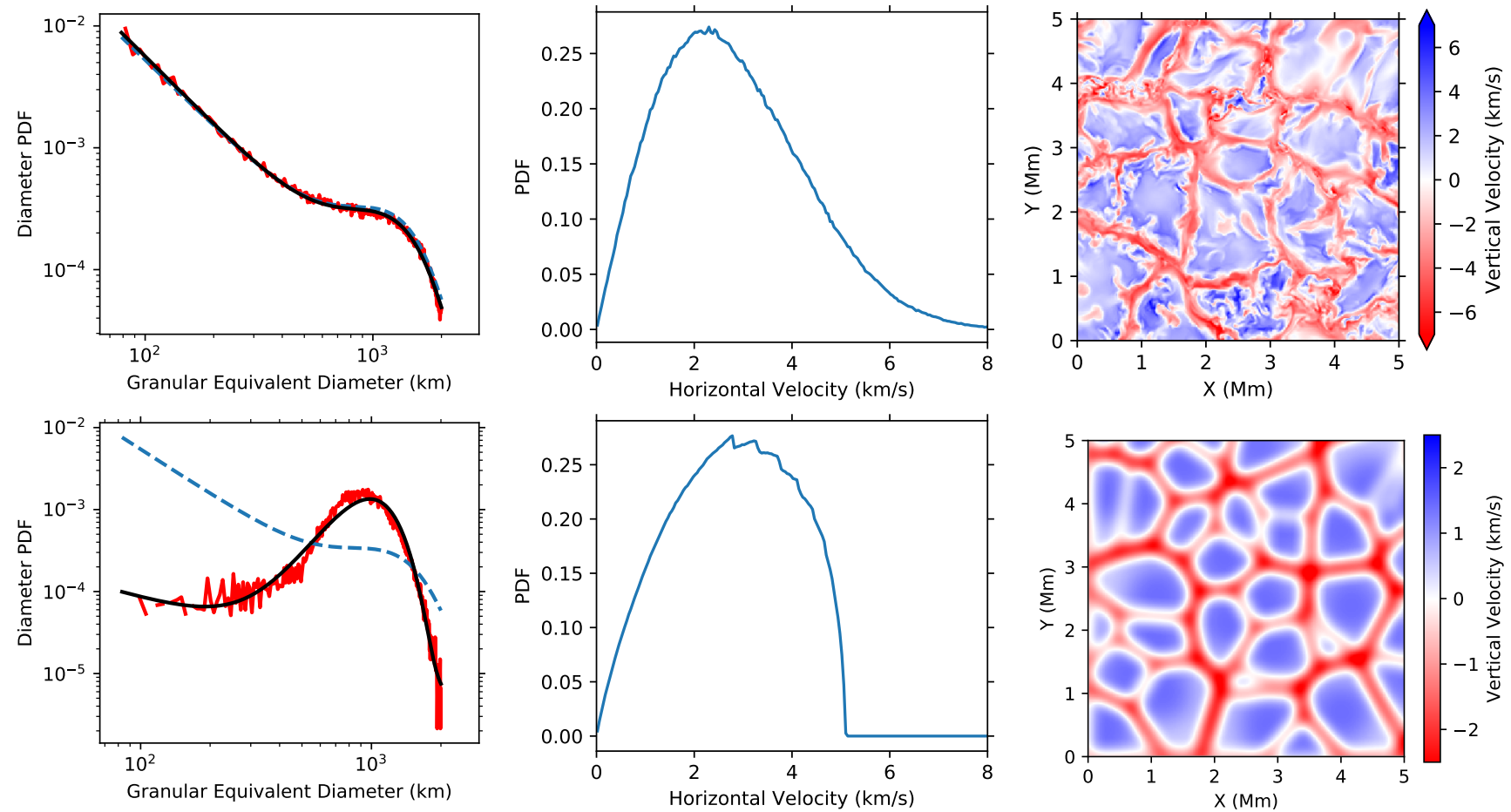

Figure 4. Comparison of MURaM (top) and ROUGH (bottom) granulation. On the left is the distribution of granule sizes. In red is the PDF of granule sizes. The dashed line is the fitted distribution of Abramenko et al. (2012) (re-normalized to this plotting range, and obscured by the other curves in the MURaM panel), while the solid curve is the same model fitted to our histogram. In the center is the histogram of horizontal velocity magnitudes at all pixels in the simulation. On the right is a sample vertical velocity map. Sizes and horizontal velocities are drawn from the full 401 frames (2.3 hours) of MURaM data, and from 1500 frames (2.5 hours) of ROUGH simulation.

area occupied by stationary inter-granular lanes. Lanes are areas of converging horizontal flow, so a cork in a lane is in a relatively stable position and will experience less motion. Further, having more granules causes each individual granule to be smaller. When a new granule emerges and rearranges the local intergranular lanes, then, the lanes will be shifted by a smaller distance and any displaced corks will be dragged along for a shorter distance before being in a stable location again. An increase in the granule scale size pushes the maximum of the radial velocity function into the region affected by the Gaussian smoothing, thus extending the low-horizontal-velocity region from the center of granules to the edges. This means that when the locations of the lanes changes, the granule-edge, horizontal flows that push corks along with the moving lane are weaker, and so corks move more slowly.

The analysis in this paper is of 3072 independent ROUGH simulation runs, with one cork per run. We use a spatial resolution of $50 \mathrm{~km} / \mathrm{pixel}$, a time step of 2 seconds, a simulation duration of 167 minutes, and a granule emergence rate of $5.9 \times 10^{-9} \mathrm{~km}^{-2} \mathrm{~s}^{-1}$.

\section{RESULTS}

\subsection{Granulation}

Here we present a comparison of the granulation patterns in ROUGH and MURaM as a validation of the ROUGH approach. In Figure 4, we compare the distribution of granule sizes between the two simulations and the observational values. The observational values, from Abramenko et al. (2012), draw on a field of view comparable to the MURaM simulation domain and a diffraction-limited resolution of $77 \mathrm{~km}$. Abramenko et al. (2012) use intensity thresholding to separate granules from intergranular lanes. As described in Section 2.2, we instead use a $V_{z}=0$ threshold, which provides more straightforward granule segmentation.

Observed granule sizes are fit by the sum of a Gaussian distribution of large granules and a power law distribution of small granules (Abramenko et al. 2012), thought to reveal the presence of two distinct populations of granules (as in Hirzberger et al. 1997). We find that the MURaM distribution reproduces very well the observational distribution. However, the ROUGH distribution contains only the Gaussian portion. This suggests that the small granules with a power-law size distribution have their origins in the turbulent phenomena not included in the ROUGH model, whereas the Gaussian-distributed large granules are produced by the large-scale, convective structures of the photosphere. 


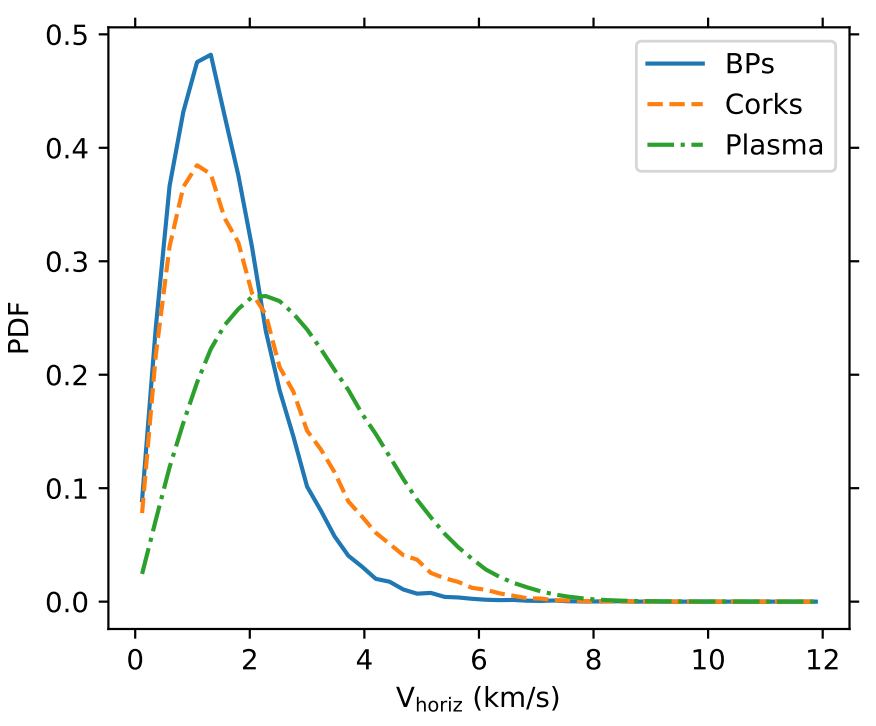

Figure 5. Horizontal velocity histogram for bright points, corks, and plasma in the MURaM data. Plasma velocity is sampled at every pixel in one frame, whereas the bright pointand cork distributions use the full-length simulation. The largest value in each histogram is $8.97 \mathrm{~km} \mathrm{~s}^{-1}$ for bright points, $10.8 \mathrm{~km} \mathrm{~s}^{-1}$ for corks, and $11.7 \mathrm{~km} \mathrm{~s}^{-1}$ for the plasma.

Figure 4 also compares the distribution of horizontal velocities in the two simulations. While the shape of the ROUGH distribution is set by the radial velocity functions (Equations $1 \& 2$ ), the horizontal scaling is set by the matching of integrated power spectra. This produces good agreement between the two horizontal velocity distributions. Notable, however, is the hard cutoff at $V_{\max }$ in the ROUGH plot, whereas the MURaM plot has a high-velocity tail due to turbulent regions. Figure 4 finally compares two granulation images, mapped in terms of vertical velocity, in which the qualitative agreement is good (aside from the presence of turbulence).

\subsection{Bright Point Statistics}

Applying the bright-point identification algorithm described in Section 2.2, we find a total of 3,185 bright points meeting all our size and lifetime thresholds in the MURaM simulation data over the simulation time of 2.3 hours (401 snapshots). Of these thresholds, the required lifetime of at least 1.6 minutes has the largest effect. 35 out of every 36 identified bright points are removed by this criterion. (See the steep lifetime distribution in Figure 6.) Of the remaining 3,185 bright points, 9 were the result of mergers, 34 ended in a merger, 37 were the product of a fragmenting bright point, and 56 ended by fragmenting.

In Figure 5, we show the distribution of observed horizontal velocities for bright points, corks, and the plasma flow. Cork velocities are lower than plasma velocities. This is consistent with the fact that corks in a high-velocity region will travel at high velocity and quickly leave, while corks in a low-velocity region will linger; low-velocity regions are therefore preferentially sampled by corks. Bright-point velocities may be susceptible to contributions from jitter in the centroid location caused by, e.g., bright-point shape changes or variation in the identified edge of the bright point. We note here that bright-point velocities are typically lower than plasma or cork velocities, suggesting this is not a strong effect. In the Appendix, we show more evidence that this effect appears to be insignificant in our data.

In Figure 6, we show selected distributions of brightpoint lifetimes, intensities, sizes, and vertical magnetic field strengths. The distributions of bright-point size, vertical flux, and intensity all cluster near a central value (respectively, $109 \mathrm{~km}, 1.25 \mathrm{kG}$, and $2.7 I_{0}$ ). The bright-point lifetime, however, shows a sharp decay from the minimum lifetime threshold (1.6 minutes), with nearly all bright points falling below a 5-minute lifetime. The mean bright-point lifetime (for bright points living longer than $1.6 \mathrm{~min}$ ) is $2.7 \mathrm{~min}$, while the maximum is $14.5 \mathrm{~min}$. This mean is comparable to the mean lifetime of $\sim 3 \mathrm{~min}$ for the longer-lived granule population in Del Moro (2004), indicating that bright points evolve on the same timescale as the granulation pattern. Our mean bright-point lifetime is notably shorter than the 9.33 minutes of Berger et al. (1998). However, they take steps to track bright points through merging and splitting as well as through short ( $\sim 40 \mathrm{~s}$ ) disappearances of bright points. We do not attempt to match these capabilities, as doing so would complicate the calculation of power spectra.

Neither bright-point lifetime nor bright-point velocity show a strong correlation with any other measured value. This may be expected for lifetime due to our inability to track bright points through splitting and merging events, meaning that our reported lifetimes do not all correspond to the true lifetimes of bright points, and due to the fact that bright points themselves are only one stage in the constant evolution of magnetic flux. The non-correlation may also be expected for velocity, since bright-point motion is controlled by external forces. However, strong correlations emerge between bright-point intensity, size, and magnetic field strength. We use for each bright point the maximum size and the $95^{\text {th }}$ percentile pixel value value for intensity and field strength in order to capture the value when the bright point is most fully emerged and vertical but to avoid outlier values. We find a correlation coefficient of 0.59 between intensity and $\left|B_{z}\right|, 0.44$ between size and $\left|B_{z}\right|$, and 0.62 between size and intensity. These correlations are all statistically significant and reasonably suggest that a stronger flux concentration will produce a larger, brighter bright point (a conclusion supported observationally, e.g. Ji et al. 2016).

The correlations we find between bright-point lifetime and both diameter and velocity differ from those found in Yang et 

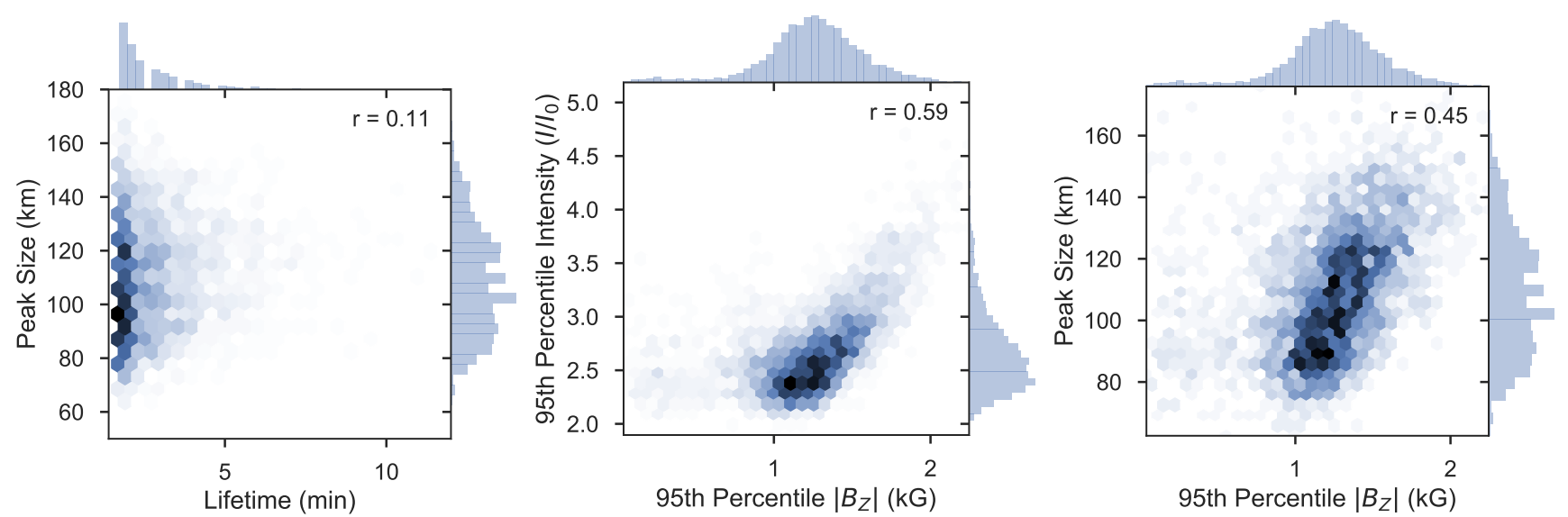

Figure 6. Distributions of bright-point statistics in the MURaM data. "Size" is an equivalent diameter. The $95^{\text {th }}$ percentiles are computed from the values at all pixels identified as within a bright point. Marginalized distributions are shown along the edges of the plots. Shown for each distribution is the correlation coefficient; each has a two-sided p-value $<10^{-5}$.

al. (2014). These Hinode/SOT observations yielded a correlation coefficient between lifetime and size to be +0.83 (contrasted with our value of +0.01 for mean values and +0.1 for peak values) and between lifetime and velocity to be -0.49 (contrasted with our -0.09 for mean values and +0.14 for peak values). The large differences here are likely due to a difference in approach. Bright-point lifetimes are discrete multiples of the imaging cadence, and for each lifetime value, Yang et al. (2014) calculate a mean value of diameter and velocity among all bright points of that lifetime and use these mean values to perform a linear regression. In contrast, we use our full dataset with all its variation. Using average values collapses the data to a mean trend line, which risks artificially enhancing the resulting correlation coefficient. We believe that using the full set of data more accurately represents the level of variation in the data (and, therefore, how well one measurable can predict another).

\subsection{Power Spectra}

Following the literature (van Ballegooijen et al. 1998; Cranmer \& van Ballegooijen 2005; Chitta et al. 2012), we compute power spectra from velocity sequences via the Wiener-Khinchin theorem, which provides a power spectrum as the Fourier transform of the autocorrelation of a data sequence. While perhaps a roundabout approach ${ }^{1}$, this allows individual bright-point velocity sequences to be more straightforwardly averaged together. Since velocity sequences of different lengths are produced, their direct Fourier transforms (and those transforms' absolute-square) have different ranges and spacings in frequency, making a more direct averaging non-trivial. But as long as different velocity

\footnotetext{
1 In fact, this is the reverse of the usual application of the WienerKhinchin theorem, which is often used as an efficient way to calculate an autocorrelation by way of a Fourier transform, an absolute-square, and an inverse Fourier transform.
}

sequences have the same temporal spacing, their autocorrelations will have the same spacing. This allows averaging to be performed on the autocorrelations (analytically equivalent to averaging the final power spectra), which is computationally simpler.

For the MURaM bright points, we calculate power spectra of the finite-difference velocities of the intensity-weighted centroids of bright points. Our observational comparison, Chitta et al. (2012), present their power spectrum and autocorrelation function as fitted, analytical functions. In this section, we present the power spectrum produced by discretely sampling the Chitta et al. (2012) autocorrelation function at its observational cadence of $5 \mathrm{~s}$ out to $\pm 105 \mathrm{~s}$ (the range used for their fit to the autocorrelation) and Fourier transforming, to provide a more direct comparison to our own power spectra.

Power spectra for observations, MURaM corks, MURaM bright points, and ROUGH corks are shown in Figure 7. ROUGH is configured so that the integrated power matches the integrated MURaM cork power.

At low frequencies, the ROUGH spectrum is similar to the MURaM cork spectrum, whereas at high frequencies it drops off precipitously. This reflects the similarity between the two simulations of the large-scale granulation patterns, which cause low-frequency movement, and the absence in ROUGH of small-scale turbulence (e.g. vortex flows, see Giagkiozis et al. 2017), which drive high-frequency motion. This comparison also suggests that these two phenomena are the dominant drivers of motion in their respective frequency regimes.

The MURaM cork spectrum shows more low-frequency power than the MURaM bright points, suggesting that perhaps corks are more free to drift in steady flows than are bright points, but the two spectra converge at high frequencies, suggesting that they respond similarly to rapid varia- 
tion in plasma flow. Finally, both MURaM analyses show a consistent enhancement in power over the Chitta data for the frequencies of overlap, quantified later in this section.

The integral of the power spectrum is equal to the variance of the velocity data (via Parseval's theorem). These integrated values are shown in Table 1 . We report both the integral of each spectrum over its full extent and the integral over the frequency range covered by all four spectra $(0.005$ $\mathrm{Hz} \leq \mathrm{f} \leq 0.02 \mathrm{~Hz}$ ). The former represents a spectrum's lower bound on the "true" total power, unconstrained by limited frequency coverage, while the latter allows spectra to be compared over a comparable domain. These numbers reveal more motion and variation for MURaM corks than for bright points, and as noted above, we find a $91 \%$ increase in total power measured in the frequencies of overlap for MURaM bright points versus observed bright points. This increase in motion, in both the power spectra and the integrated spectra, might be explained in part by the calibration offset applied by Chitta et al. (2012), a step which could tend to damp out some real motion in addition to spurious motion and which is not needed in simulation analysis. It might also suggest there is, in fact, more power to be detected with higher-quality observations. Improvements in spatial resolution and sensitivity will make bright-point detection and centroid identification more reliable and make smaller bright points visible, both enabling more robust statistics. Verification of the presence of unmeasured power must wait until DKIST comes online, currently scheduled for 2019. The Visible Broadband Imager on DKIST will have a diffraction-limited, G-band resolution of $\sim 16 \mathrm{~km}$ (comparable to these MURaM simulations) and a 3 second cadence (Elmore et al. 2014), improving on both the $\sim 160 \mathrm{~km}$ resolution of the Hinode SOT observations of Yang et al. $(2014,2015)$ and the $\sim 100 \mathrm{~km}$ resolution and 5 second cadence of the SST observations of Chitta et al. (2012).

Both the MURaM and Chitta et al. (2012) power spectra shown in Figure 7 indicate power-law behavior with slopes of order $f^{-1}$ between $10^{-3}$ and $10^{-2} \mathrm{~Hz}$ (see Table 1). This is a much flatter spectrum than the presumed exponential drop-off associated with random-walk-like motions like those found from earlier measurements of bright points (e.g., van Ballegooijen et al. 1998; Cranmer \& van Ballegooijen 2005). However, whether this points to the presence of an active turbulent cascade (Petrovay 2001) in the photosphere is still not known. Reduced MHD turbulence simulations for solar flux tubes (van Ballegooijen et al. 2011; Woolsey \& Cranmer 2015) showed that low-frequency random-walk motions rapidly produce a steep power-law spectrum $(P \sim$ $\left.f^{-4.5}\right)$ in the photosphere. This may be modified for the flatter bright-point driving spectrum found in this work. There is a long history of interplanetary $f^{-1}$ fluctuations being interpreted as fossil remnants of oscillations that map down to the solar surface (Matthaeus \& Goldstein 1986; Velli et al. 1989;

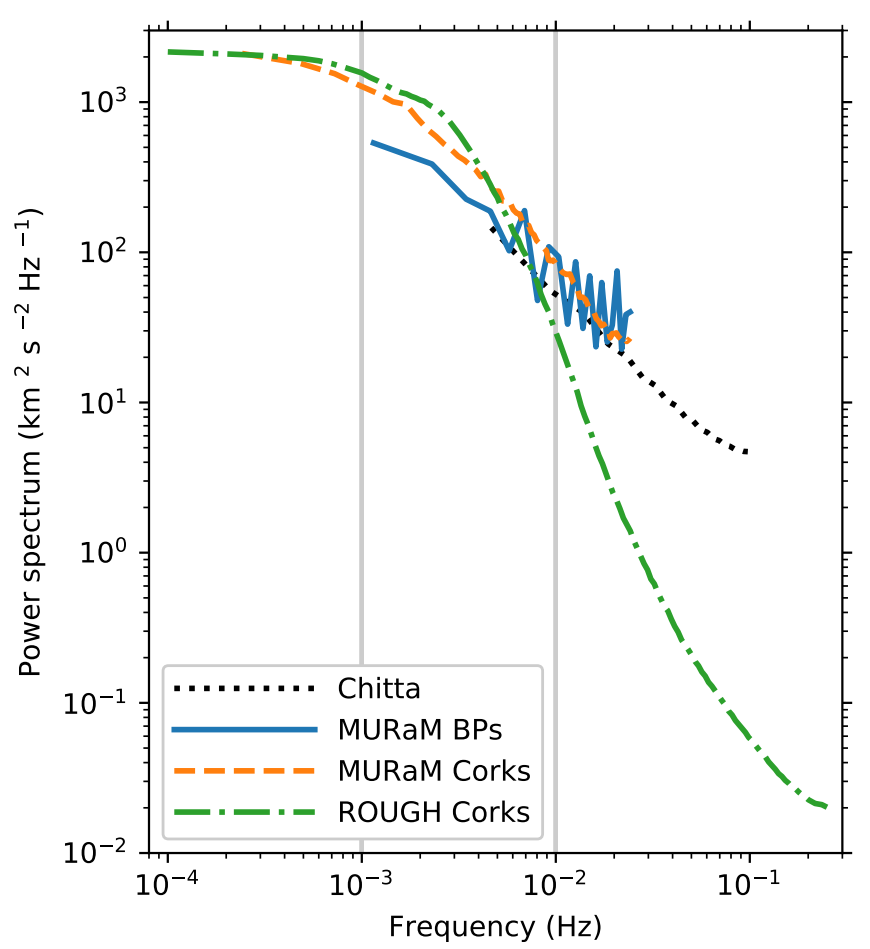

Figure 7. All power spectra mentioned in this paper. The MURaM cork curves are those of Section 2.2. The MURaM curve is from our bright point tracking approach described in Section 2.2. The Chitta curve is from the observations of Chitta et al. (2012). The ROUGH curve comes from our simulations, described in Section 3.1. Gray vertical lines mark the frequencies of the slope measurements in Table 1.

Dmitruk \& Matthaeus 2007; Verdini et al. 2012). However, those fluctuations occur at even lower frequencies than those studied in this paper (e.g., $f<10^{-4} \mathrm{~Hz}$ ) and their physical origin is still being debated.

\section{DISCUSSION AND CONCLUSIONS}

The goal of this paper was to better understand the horizontal motions of photospheric intergranular bright points. We characterized this motion using simulations that exceed present observational capabilities, and we investigated which aspects of the granular flows drive this motion.

Using high-resolution simulations, we found an increase of $91 \%$ over observations in the power spectrum of brightpoint motion at all frequencies of overlap. This suggests the possibility that the limitations of current observations underrepresent the motion of bright points - an idea to be tested by DKIST observations. The power spectrum of MURaM bright points is only negligibly affected when blurring the simulation output to an observational resolution. However, the expected increase in signal-to-noise ratio for DKIST observations also plays a significant role in improving brightpoint tracking reliability. 
Table 1. Properties of the spectra in Figure 7.

\begin{tabular}{lcccc}
\hline \hline \multirow{2}{*}{ Spectrum } & \multicolumn{2}{c}{ Integrated Power $\left(\mathrm{km}^{2} \mathrm{~s}^{-2}\right)$} & \multicolumn{2}{c}{ Slope at } \\
& over all frequencies & $0.005 \mathrm{~Hz} \leq \mathrm{f} \leq 0.02 \mathrm{~Hz}$ & $0.001 \mathrm{~Hz}$ & $0.01 \mathrm{~Hz}$ \\
\hline Chitta & 1.87 & 0.56 & $\ldots$ & -1.25 \\
MURaM BPs & 3.46 & 1.07 & $\ldots$ & -1.27 \\
MURaM Corks & $5.42^{\mathrm{a}}$ & 1.21 & -0.37 & -1.59 \\
ROUGH Corks & $5.42^{\mathrm{a}}$ & 0.57 & -0.45 & -3.45
\end{tabular}

${ }^{a}$ ROUGH's $V_{\max }$ value is set so its corks' integrated spectrum matches that of the MURaM corks.

We compared the power spectra of passive tracers in MURaM flows and ROUGH's simplified, turbulence-free flows. This comparison suggested that low-frequency motion of passive tracers (and thus bright points) is driven by longterm granular evolution, while high-frequency motion is due to turbulent effects.

We found that the power spectra of bright-point motion and passive-tracer motion are very similar at high frequencies, but passive tracers display about 2.5 times more power at low frequencies. One possible explanation for this is that the passive tracers move according to the plasma flow field at the $\tau=1$ surface only, whereas bright points are pushed at multiple depths along the flux tube. Any depth dependence to the horizontal plasma velocity will result in competing forces on the flux tube, producing muted bright-point motion. That this difference is seen only at low frequencies may suggest that rapid changes to the flow field (producing high-frequency motion) are either coherent across the relevant depths or are sufficiently strong at one depth to dominate over the flows at other depths, whereas long-term flows have more depth dependence and so produce net smaller effects on bright points.

We found that MURaM effectively reproduces the granule size distribution of the observations in Abramenko et al. (2012). However, of this two-component distribution, ROUGH reproduces only one component-the Gaussiandistributed, large granules. We proposed that the two components to the granule size distribution represent the large convective cells and the small, turbulent features. Separately, Del Moro (2004) finds that granule lifetimes are best fit by a two-exponential model, finding a different fit for the $>5$ minute and $<5$ minute lifetimes. We speculate that these two fits match the two classes in the granule size distribution-the small, turbulent features correspond to the short-lived features, and the large-scale convective features correspond to the long-lived features. Indeed, Del Moro (2004) finds a correlation between granule lifetime and size that is positive for short-lived granules, but which flattens for lifetimes over $\sim 8$ minutes.

We found that bright-point size, brightness, and magnetic flux are all positively correlated (with $r \sim 0.5$ ). This can be made sense of, remembering that bright points are bright because a reduction in gas density lowers the $\tau=1$ surface to hotter, brighter regions. A larger-diameter flux tube will tend to allow observations deeper into the tube before bending cuts off the line of sight, while stronger magnetic fields will produce lower gas pressure and density in the flux tube that allows a clearer view deeper into the tube.

It is worthwhile to use the results found above to investigate some properties of Alfvén fluctuations that propagate up into the corona and give rise to dissipative heating. Following Cranmer \& van Ballegooijen (2005), it is possible to estimate the net upward energy flux $F$ of Alfvén waves given the properties at the photosphere,

$$
F=\rho\left\langle v_{\perp}^{2}\right\rangle V_{\mathrm{A}}\left(\frac{1-\mathcal{R}}{1+\mathcal{R}}\right)
$$

where we assume a photospheric density $\rho=2 \times 10^{-7}$ $\mathrm{g} \mathrm{cm}^{-3}$ and typical bright point field strength $B=1500$ $\mathrm{G}$ in order to estimate the Alfvén speed $V_{\mathrm{A}}=B / \sqrt{4 \pi \rho}$, and the velocity variance $\left\langle v_{\perp}^{2}\right\rangle$ is the integrated power given in Table 1. The above expression also depends on the reflection coefficient for wave energy $\mathcal{R}$, which Cranmer \& van Ballegooijen (2005) found to be approximately 0.90 below the transition region.

Note that Equation (4) gives the energy flux inside a vertically oriented bright-point flux tube. However, in the corona, these flux tubes are expected to broaden out to eventually fill the entire volume. Thus, a more relevant energy flux for coronal heating is the surface-averaged value $\langle F\rangle \approx f_{*} F$, where $f_{*}$ is the photospheric filling factor of bright points, which we treat as a simple, unweighted area fraction. In the MURaM simulation analyzed above, $f_{*}=0.00474$ for all identified bright points, including those with lifetimes below 1.6 minutes that did not yield useful velocity information. Combining the above quantities, we find $\langle F\rangle \approx 860 \mathrm{~W} \mathrm{~m}^{-2}$ for the Chitta et al. (2012) integrated power, $1590 \mathrm{~W} \mathrm{~m}^{-2}$ for the power in MURaM bright points, and $2490 \mathrm{~W} \mathrm{~m}^{-2}$ for the power in MURaM cork motions. These values are well above the classical Withbroe \& Noyes (1977) empirical requirements for coronal heating in the quiet $\operatorname{Sun}\left(300 \mathrm{~W} \mathrm{~m}^{-2}\right)$ and coronal holes $\left(800 \mathrm{~W} \mathrm{~m}^{-2}\right)$, indicating that only a frac- 
tion of the wave energy flux needs to be dissipated as heat in these regions.

One aspect not considered in this work is the impact of long chains of connected bright points seen in regions of strong background magnetic flux (Dunn \& Zirker 1973; Sánchez Almeida et al. 2004). These features were considered by van Ballegooijen et al. (1998), but they generally require a different approach than that used in this paper for small, isolated bright points. Differences may be expected between the dynamics of the isolated and the extended types of bright points, due to both interactions between flux elements in extended structures, and the observed trend for bright points to move more slowly in regions of increased magnetic flux (Ji et al. 2016; Yang et al. 2016).

While the transverse bright-point motion studied in this paper is useful, it does not tell the complete story. MHD waves can also be excited both inside and outside the brightpoint flux tubes by compressive and longitudinal fluctuations. This fact will become more prominent when DKIST turns traditionally-point-like bright points into resolved structures in which the motions necessary to excite these additional modes will become observable. In fact, as discussed in the Appendix, the ability to track higher-order motion may become necessary. We plan to study these wave modes in future work. Beyond MHD waves, bright-point motion can also be analyzed in bulk, looking at the diffusive statistics of the bright points (e.g. Abramenko et al. 2011; Ji et al. 2016; Agrawal et al. 2017; Jafarzadeh et al. 2017).

Future efforts at bright point tracking may include algorithm refinements. Berger et al. (1998) differ from our approach in their ability to sensibly track bright points through merging and splitting events, whereas we simply consider the products of such events (which are fortunately rare in our MURaM data set) to be newly-found bright points. Their algorithm is also resilient to bright points which disappear for up to a few frames before reappearing. However, this improvement may become unnecessary with the high cadence and sensitivity of DKIST data. Algorithmic improvements to improve robustness or detection quality might also be adapted from the recommendations of DeForest et al. (2007), benefiting from existing efforts in large-scale feature detection.

The authors warmly thank Matthias Rempel for sharing the results of his MURaM simulations, and Mark Rast and Piyush Agrawal for discussing with us the results of their own work and inspiring the contents of this paper's appendix. SJV thanks Craig DeForest and Derek Lamb for a productive summer spent learning many coding techniques used in this analysis. This work was supported by NSF grant 1613207 (AAG). SRC's work was also supported by NASA grants NNX15AW33G and NNX16AG87G, NSF grant 1540094 (SHINE), and start-up funds from the Department of Astrophysical and Planetary Sciences at the University of Colorado Boulder. Portions of this work utilized the RMACC Summit supercomputer, which is supported by the National Science Foundation (awards ACI-1532235 and ACI-1532236), the University of Colorado Boulder, and Colorado State University. The Summit supercomputer is a joint effort of the University of Colorado Boulder and Colorado State University. This work made extensive use of the Scientific Python ecosystem and NASA's Astrophysics Data System.

\section{APPENDIX}

\section{A. CENTROID JITTER}

The tracking of centroids in identified features is susceptible to jitter in the centroid motion due to the effect of feature shape changes on the computed centroid location. Whether the shape change is due to twisting or compression of the bright point, the merging of sub-resolution bright points with the tracked bright point, or is just an artifact of the tracking routine due to changes in the identified edge of the bright point, a change in shape can produce additional centroid motion beyond idealized, rigid-body advection of the bright point. This additional motion may represent real motion of magnetic field lines and a source of higherorder MHD waves. However, in the context of tracking bright-point centroids as representative of the flux tube's bulk motion (the context of this and most other papers tracking bright points as MHD wave sources), this additional motion is spurious. Centroid tracking becomes vulnerable to this effect only when increased spatial resolution changes bright points from points into welldefined features - i.e. high-resolution simulations and DKIST observations. This effect is explored more fully in Agrawal et al. (2017); here we attempt to constrain the influence of this effect on power spectra such as those presented in this paper.

Between two consecutive Frames 1 and 2, a bright point will have measured areas $A_{1}$ and $A_{2}$, with a size change $\Delta A \equiv$ $A_{2}-A_{1}$. In this section we use a simple model of the Frame 1 bright point as a square of side length $\sqrt{A_{1}}$ in Frame 1 , and the bright point in Frame 2 as a rectangle of side lengths $\sqrt{A_{1}}$ and $\sqrt{A_{1}}+\ell$, where $\ell=\Delta A / \sqrt{A_{1}}$ so that the rectangle's area is $A_{1}+\Delta A=A_{2}$. This is an intermediate case for the effect of a size change $\Delta A$ on the centroid location, where the extremes are uniform, radial expansion having no effect on the centroid, and the addition of a long and thin filament to the shape of Frame 1 
having an extreme effect on the centroid. In this model, and assuming no advection of the bright point, the unweighted centroid will move a distance of $\Delta x=\Delta A /\left(2 \sqrt{A_{1}}\right)$ along the major axis of the rectangle.

We calculated $\Delta x$ from measured areas for each bright point and for each timestep in our 20-second-cadence MURaM simulation. We did the same after applying our tracking routine to a 2-second-cadence MURaM run (without the $30 \mathrm{G}$ net injected flux of the 20-second-cadence run; see Rempel (2014); Agrawal et al. (2017)). While this 2-second-cadence run has a smaller spatial domain $(6 \times 6 \mathrm{Mm}$, versus the $24.5 \times 24.5 \mathrm{~km}$ of the 20 -second-cadence simulation used in this paper $)$ and thus provides fewer bright points and poorer statistics, it allows us to investigate how DKIST measurements might be affected by this centroid jitter.

To simulate the contribution of centroid jitter to the motion of a bright point, we then average the power spectra of every 30-sample sequence of $\Delta x$ values. These spectra are shown in Figure 8. The jitter spectra for the two different cadences produce a nearly-continuous line. The spectrum for the 20 -second-cadence data exceeds the jitter spectrum while showing a consistent slope of opposite sign. The spectrum for the 2 -second-cadence data, however, coincides with the 20 -second-cadence spectrum for low frequencies, but levels off and even takes on a positive slope for the highest frequencies. In the mid- to high-frequency regime, this spectrum suggestively takes the shape of the jitter spectrum.

We interpret this to mean that, at a 20-second-cadence, the jitter in a bright point's centroid is small compared to the advective motion between frames, and the analysis in this paper is safe from misinterpreting jitter as bulk advection. However, the additional frequencies probed by the 2 -second-cadence data appear to be dominated by jitter rather than advection. We base this on the similarity in slope between the bright-point and jitter spectra and attribute the factor of $\sim 2$ difference in magnitude to the simplicity of our jitter model. This effect may not have been visible in the high-cadence Chitta et al. (2012) observations due to their spatial resolution rendering unresolvable the shape changes that give rise to this jitter. However, we anticipate both high cadence and high spatial resolution in DKIST data. Therefore, analysis of MHD wave excitation by bright points observed by DKIST must take a more advanced approach or it will risk assigning the energy in higher-order modes to simple, bulk advection.

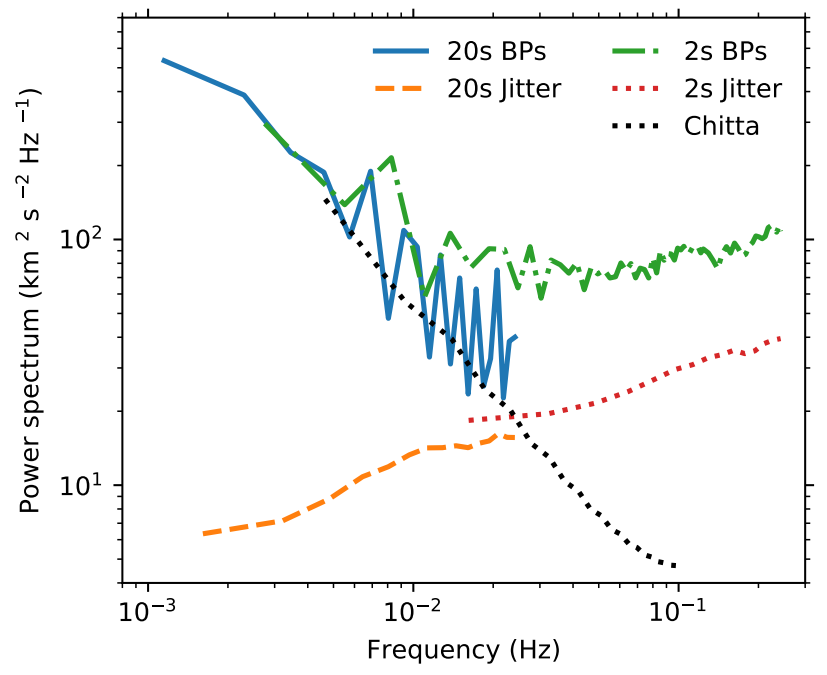

Figure 8. Power spectra of the total bright point (BP) motion and the modeled contribution to that motion of centroid jitter for bright points in 20-second- and 2-second-cadence MURaM runs. The 20-second-cadence bright point spectrum is the same as is shown in Figure 7, and the observational Chitta et al. (2012) spectrum of that figure is also reproduced to facilitate comparison.

\section{REFERENCES}

Abramenko, V. I., Carbone, V., Yurchyshyn, V., et al. 2011, ApJ, 743,133

Abramenko, V. I., Yurchyshyn, V. B., Goode, P. R., Kitiashvili,

I. N., \& Kosovichev, A. G. 2012, ApJL, 756, L27

Agrawal, P., Rast, M., Gošić, M., Bellot Rubio, L., \& Rempel, M.

2017, in preparation
Berger, T. E., Löfdahl, M. G., Shine, R. S., \& Title, A. M. 1998, ApJ, 495, 973

Berger, T. E., \& Title, A. M. 1996, ApJ, 463, 365

Carlsson, M., Stein, R. F., Nordlund, Å., \& Scharmer, G. B. 2004, ApJL, 610, L137

Chitta, L. P., van Ballegooijen, A. A., Rouppe van der Voort, L., DeLuca, E. E., \& Kariyappa, R. 2012, ApJ, 752, 48 
Cranmer, S. R., \& van Ballegooijen, A. A. 2005, ApJS, 156, 265

DeForest, C. E., Hagenaar, H. J., Lamb, D. A., Parnell, C. E., \& Welsch, B. T. 2007, ApJ, 666, 576

Del Moro, D. 2004, A\&A, 428, 1007

Dmitruk, P., \& Matthaeus, W. H. 2007, PhRvE, 76, 036305

Dunn, R. B., \& Zirker, J. B. 1973, SoPh, 33, 281

Elmore, D. F., Rimmele, T., Casini, R., et al. 2014, Proc. SPIE, 9147, 914707

Feng, S., Ji, K.-F., Deng, H., Wang, F., \& Fu, X.-D. 2012, Journal of Korean Astronomical Society, 45, 167

Giagkiozis, I., Fedun, V., Scullion, E., \& Verth, G. 2017, A\&A, submitted, arXiv:1706.05428

Hirzberger, J., Vázquez, M., Bonet, J. A., Hanslmeier, A., \& Sobotka, M. 1997, ApJ, 480, 406

Jafarzadeh, S., Solanki, S. K., Cameron, R. H., et al. 2017, ApJS, 229,8

Ji, K.-F., Xiong, J.-P., Xiang, Y.-Y., et al. 2016, Research in Astronomy and Astrophysics, 16, 78

Keys, P. H., Mathioudakis, M., Jess, D. B., et al. 2011, ApJL, 740, L40

Lemmerer, B., Hanslmeier, A., Muthsam, H., \& Piantschitsch, I. 2017, A\&A, 598, A126

Matthaeus, W. H., \& Goldstein, M. L. 1986, Physical Review Letters, 57, 495

Nisenson, P., van Ballegooijen, A. A., de Wijn, A. G., \& Sütterlin, P. 2003, ApJ, 587, 458

Nordlund, Å., Stein, R. F., \& Asplund, M. 2009, Living Reviews in Solar Physics, 6, 2

Oba, T., Iida, Y., \& Shimizu, T. 2017, ApJ, 836, 40

Petrovay, K. 2001, SSRv, 95, 9
Puschmann, K. G., Ruiz Cobo, B., Vázquez, M., Bonet, J. A., \& Hanslmeier, A. 2005, A\&A, 441, 1157

Rempel, M. 2014, ApJ, 789, 132

Roudier, T., Rieutord, M., Malherbe, J. M., et al. 2012, A\&A, 540, A88

Sánchez Almeida, J., Márquez, I., Bonet, J. A., Domínguez Cerdeña, I., \& Muller, R. 2004, ApJL, 609, L91

Simon, G. W., \& Weiss, N. O. 1989, ApJ, 345, 1060

Steiner, O., Hauschildt, P. H., \& Bruls, J. 2001, A\&A, 372, L13

Tritschler, A., Rimmele, T. R., Berukoff, S., et al. 2016, Astronomische Nachrichten, 337, 1064

Utz, D., Hanslmeier, A., Muller, R., et al. 2010, A\&A, 511, A39 van Ballegooijen, A. A., Nisenson, P., Noyes, R. W., et al. 1998, ApJ, 509, 435

van Ballegooijen, A. A., Asgari-Targhi, M., Cranmer, S. R., \& DeLuca, E. E. 2011, ApJ, 736, 3

Velli, M., Grappin, R., \& Mangeney, A. 1989, Physical Review Letters, 63, 1807

Verdini, A., Grappin, R., Pinto, R., \& Velli, M. 2012, ApJL, 750, L33

Vögler, A., Shelyag, S., Schüssler, M., et al. 2005, A\&A, 429, 335

Withbroe, G. L., \& Noyes, R. W. 1977, ARA\&A, 15, 363

Woolsey, L. N., \& Cranmer, S. R. 2015, ApJ, 811, 136

Yang, Y.-F., Lin, J.-B., Feng, S., et al. 2014, Research in Astronomy and Astrophysics, 14, 741-752

Yang, Y.-F., Qu, H.-X., Ji, K.-F., et al. 2015, Research in Astronomy and Astrophysics, 15, 569

Yang, Y., Li, Q., Ji, K., et al. 2016, SoPh, 291, 1089 